\title{
The theory and practice of workload control: A research agenda and implementation strategy
}

\author{
Mark Stevenson $^{\mathrm{a}, *}$, Yuan Huang ${ }^{\mathrm{a}, 1}$, Linda C. Hendry ${ }^{\mathrm{a}, 2}$, Erik Soepenberg ${ }^{\mathrm{b}, 3}$ \\ a Department of Management Science, Lancaster University Management School, Lancaster University, LA1 4YX UK \\ ${ }^{\mathrm{b}}$ University of Groningen, Department of Operations, Faculty of Economics and Business University of Groningen, PO Box 800,9700 AV Groningen, The Netherlands
}

\section{A R T I C L E I N F O}

\section{Article history:}

Received 24 August 2010

Accepted 15 February 2011

Available online 23 February 2011

\section{Keywords:}

Workload Control

Implementation

Make-To-Order

Small and Medium sized Enterprise

Production Planning and Control

\begin{abstract}
A B S T R A C T
The Workload Control (WLC) concept is one of few Production Planning and Control (PPC) solutions appropriate for Make-To-Order (MTO) companies yet its successful implementation is an enduring challenge. Most implementations reported are in large organisations yet it has been argued that WLC is particularly suitable for Small and Medium sized Enterprises (SMEs) with limited financial resources. Moreover, previous studies do not adequately describe the process through which implementation success was achieved. In this paper, data collected through semi-structured face-to-face interviews with key personnel from 41 companies is presented in order to build up a body of evidence on the characteristics of MTO SMEs that affect WLC implementation. The data paints a complex picture of MTO production and suggests that research is likely to be required to move theory closer to practice and organisational change to move practice closer to theory. The former is recommended where processes perform well or appear difficult to change; the latter is recommended where fundamental WLC principles are involved. Two outputs emerge from the study: (1) a research agenda for refining the WLC concept to improve alignment between theory and practice, covering the customer enquiry, order entry, design and engineering and order release stages; and (2) an implementation strategy, including elements of organisational change and covering pre-implementation, implementation and postimplementation stages. The characteristics of MTO SMEs identified should be used in future research to develop more realistic simulations for testing conceptual refinements while field research should apply and extend the implementation strategy presented in order to develop a more detailed roadmap for successful WLC implementation in practice.
\end{abstract}

(c) 2011 Elsevier B.V. All rights reserved.

\section{Introduction}

Workload Control (WLC) is one of few Production Planning and Control (PPC) concepts suitable for Make-To-Order (MTO) companies (Henrich et al., 2004a; Stevenson et al., 2005). It is also particularly relevant to Small and Medium sized Enterprises (SMEs) with limited financial resources (Land and Gaalman, 2009); such companies play important roles in supply chains (Hendry, 2006). Nonetheless, very few successful implementations of WLC in MTO SMEs have been described in the literature. Implementations have tended to be in large, sometimes atypical companies; for example, the implementation of WLC in a large

\footnotetext{
* Corresponding author. Tel.: +44 1524593847.

E-mail addresses: m.stevenson@lancaster.ac.uk (M. Stevenson), y.huang10@lancaster.ac.uk (Y. Huang), 1.hendry@lancaster.ac.uk (L.C. Hendry), g.d.soepenberg@rug.nl (E. Soepenberg).

1 Tel.: +441524593707.

2 Tel.: + 441524593841 .

3 Tel.: +310503638317.
}

manufacturer of plastic leaves (Bechte, 1988). When researchers have attempted to implement WLC in MTO SMEs, they have encountered significant difficulties which have either delayed or halted the project (e.g., Hendry et al., 1993; Stevenson, 2006); there are two key reasons for this. First, because of a misalignment between theory and practice: WLC has been largely developed through simulations of simple systems while field researchers have encountered more complex systems; these complex systems present challenges that theoretical developments of the concept have not considered in sufficient depth, e.g., rush orders and sequence dependent set-up times. Second, because a detailed strategy to guide successful implementation has not been developed: while authors like Bechte have reported impressive reductions in lead times and Work-In-Process (WIP), they have rarely given adequate insight into how success was achieved.

Recent research has begun to identify issues affecting WLC implementation in MTO SMEs and describe how the concept must be refined to meet the needs of practitioners (e.g., Hendry et al., 2008; Stevenson and Silva, 2008). Thus far, this has been based on 
comparative analysis of two cases at-a-time, thereby limiting the generality of findings. There is a need to build up a larger body of evidence on the characteristics of MTO SMEs in order to investigate implications for the development of WLC and a strategy for its implementation. This will improve the fit between theory and practice and support more widespread adoption of WLC. This paper contributes by collecting cross-sectional data on the characteristics of MTO SMEs through semi-structured interviews in 41 companies. It uses this evidence to outline a future research agenda and propose an initial implementation strategy for WLC.

The remainder of the paper is organised as follows. Section 2 briefly introduces the WLC concept before providing a review of previous WLC implementations and issues affecting WLC implementation. Section 3 outlines the research method before Section 4 analyses evidence from the companies. Section 4 is split into three subsections; each ends by identifying contributions to the overall research agenda and implementation strategy. The research agenda and implementation strategy are summarised in Section 5 before the paper concludes with Section 6 .

\section{Literature review}

Many WLC methods are described in the literature; the most comprehensive consist of four planning stages and control a three-tiered hierarchy of workloads (the total, planned and released workload). First, the customer enquiry stage takes place between a customer making a request for quotation and an order being accepted/rejected (Kingsman et al. 1996). It includes determining whether to bid for an order and, if so, what the Due Date (DD) and price should be. A proportion of the workload of unconfirmed jobs may be incorporated in the total workload of the shop based on the probability of winning a tender (Kingsman and Mercer, 1997). Second, order entry begins with order acceptance/rejection and includes pre-production preparation for confirmed orders (e.g., checking material availability); if an order is accepted, it is incorporated in the planned workload. Third, order release, where the key decision is when to begin production; jobs are held in a pre-shop pool and considered for release, e.g., according to shortest slack or latest release date. The load of a job is compared with the current load and limits of work centres and, if one or more limits would be exceeded by releasing the job, it is retained in the pool until the next release date; otherwise, the job is released and its load contributes to the released workload of the work centres. Various order release methods have been proposed in the literature; the main difference between the approaches is how they treat the indirect load at release (see Land and Gaalman, 1998). Finally, shop floor dispatching; given the control of the above three stages, only simple dispatching rules are required and responsibility for this stage can be handled by the shop floor supervisor.

From the above it follows that key WLC decisions relate to input control, including: DD quotations; job acceptance; when to start a job (i.e., the release decision); and order progress control on the shop floor (i.e., through simple priority dispatching). In addition, WLC emphasises the use of output control through capacity adjustments. Fundamental principles underpinning WLC include: control the total input rate of work in accordance with the output rate; restrict and control the amount of work on the shop floor, and, stabilise throughput times as customers value reliability.

Section 2.1 reviews literature on the results of WLC implementation before Section 2.2 reviews research which highlights issues affecting implementation, based on pre- and postimplementation analysis. The review concludes with an overall assessment in Section 2.3. For a detailed description of WLC, see Land and Gaalman (1996) and Thürer et al. (in press).

\subsection{WLC in practice: implementation results}

The most successful applications of WLC are arguably those presented by Bechte (1988, 1994)and Wiendahl et al. (1992), where a WLC method known as Load Oriented Manufacturing Control (LOMC), featuring Load Oriented Order Release (LOOR), was implemented. This work demonstrated that WLC can improve performance in practice but made little contribution to our understanding of the implementation process. First, Bechte (1988) implemented LOMC in a large manufacturing company producing plastic leaves, reporting reductions in WIP and lead times. Bechte only briefly touched on the issue of start-up effects: it will take time to capture the current shop status and for appropriate parameters to be determined. Second, Wiendahl et al. (1992) presented two case studies where software based on LOMC had been implemented. Both were large companies: a printed circuit board manufacturer and a pump producer. Reductions in WIP and lead times were observed in both cases. Finally, Bechte (1994) presented the case of a pump producer potentially the same producer referred to in Wiendahl et al. (1992) - again noting reductions in WIP and lead times. The only implementation issues identifiable in the paper relate to software: a new software calendar had to be installed for lead time calculations and backward scheduling along with new work centre and transaction data files.

Two additional successful applications by Fry and Smith (1987) and Wiendahl (1995) provide a starting point towards an implementation strategy. First, Fry and Smith (1987) outlined a 6-stage implementation guide; however, this did not generate a comprehensive list of detailed issues that must be addressed. Second, Wiendahl (1995) also provided six steps; the first five being pre-implementation or preparatory steps: (1) manufacturing analysis; (2) manufacturing process improvement; (3) feedback accuracy improvement; (4) monitoring system; and (5) checking present manufacturing control. The sixth stage is the implementation of load oriented manufacturing control. Wiendahl (1995) also explained that companies must give up 'traditional concepts' of manufacturing. In other words, successful implementation may hinge on overcoming cultural issues within a company; however more detailed information on the process of implementing WLC, and which considers other WLC approaches than LOMC, is still required.

Research has also demonstrated that performance improvements can be achieved when WLC is embedded within existing working practices. Park et al. (1999) implemented WLC software for the customer enquiry stage in a large rotating machinery shop. This helped managers set feasible DDs but only considered the current load of the bottleneck machine; the company's existing release policy was retained. Similarly, Riezebos et al. (2003) introduced LOMC principles into a small manufacturer of corrugated cardboard packing material. An order acceptance policy was introduced with lead time reductions reported; again, the company's existing release policy was retained. Further empirical studies, where implementation success was inconclusive, have been presented, e.g., Tatsiopoulos (1983), Hendry (1989), Hendry et al. (1993), Stevenson (2006) and Silva et al. (2006). Some of these papers made more of a contribution to understanding the implementation process for WLC. For example, Hendry (1989) and Hendry et al. (1993) identify pitfalls encountered, such as selecting an inappropriate end-user for a WLC system, but without describing in detail how these pitfalls could be overcome. More recently, Silva et al. (2006) developed WLC software which 
incorporated web functionality to improve accessibility for multiple users. Stevenson and Hendry (2007) also explored webfunctionality but to integrate supply chain partners.

\subsection{WLC implementation issues: pre- and post-implementation analysis}

Two contributions to understanding issues affecting implementation - by Henrich et al. (2004a) and Land and Gaalman (2009) - focused on the pre-implementation stage. First, Henrich et al. (2004a) developed a framework for assessing the applicability of WLC to a company. Twelve best-fit indicators or characteristics were presented which could be compared against a company's characteristics to assess whether WLC was suitable; the better the match with the indicators, the more likely that WLC would be suitable. The framework focused on product and production process characteristics, such as: long routings; convergent routings (e.g., for sub-assemblies); an order book made up of a small number of large jobs; large processing times; and, tight DDs. The framework was tested on one medium-sized MTO company, demonstrating it to be a useful systematic approach for gauging the applicability of WLC. For example, in the application, set-up times were considered 'too high'; hence, they would have to be reduced before WLC could be implemented. While the paper provides a useful examination of factors affecting the suitability of WLC, the set of factors is quite narrow in scope. Moreover, assessment begins at the order review and release stage while, for MTO applicability, the customer enquiry stage is also important as the characteristics of jobs can vary greatly and DDs must be determined individually.

Second, Land and Gaalman (2009) installed a scanning system in seven MTO SMEs to collect order progress data (without implementing any PPC changes). The data was used to identify root causes of poor logistic (or delivery) performance. Analysis used throughput diagrams (Wiendahl, 1995) and order progress diagrams (Soepenberg et al., 2008), highlighting the contribution of the Design and Engineering (D\&E) stage (after order entry) to the overall lead time; in many cases, engineers did not have a clear understanding of their workload or capacity. As a result, late availability of materials was a common cause of late order completion. The authors called for more research into PPC concepts of relevance to MTO SMEs and into how pre-production processes could be incorporated into PPC concepts such as WLC. As in Henrich et al. (2004a), order acceptance was the starting point of the analysis while the authors noted the limited generality of findings from just seven cases.

Two contributions - by Stevenson and Silva (2008) and Hendry et al. (2008) - focussed on post-implementation rationalisation, reflecting on experience and identifying issues for future research. First, Stevenson and Silva (2008) presented comparative case study analysis of two implementations of the same WLC methodology (based on Hendry, 1989) in two SMEs. The companies were compared based on: demand data (e.g., demand variability and rush orders); current planning procedures (e.g., for customer enquiries); production process and shop floor characteristics (e.g., bottlenecks); product characteristics (e.g., routing length and processing times); capacity management (e.g., capacity flexibility); and technical aspects (e.g., soft/hardware availability). The paper considered how WLC was refined during the two implementations and categorised reasons for refinements into: refinements due to the time elapsed since the development of the original methodology (e.g., advances in WLC research and technology since 1989); and, refinements due to company characteristics. For the latter, refinements were split into those likely to have resulted from idiosyncratic characteristics and those which were more likely to be generic. A number of implementation challenges were also highlighted, including: meeting the data requirements of WLC and the need to increase awareness of WLC in practice. The paper provided an insight into the range of company characteristics affecting WLC but was based on just two cases. Hence, while supposedly generic refinements were proposed, the true generality of these could not be assessed.

Second, Hendry et al. (2008) conducted comparative case study analysis of issues that arose while implementing WLC in two SMEs: one capital goods manufacturer (MTO company); and one precision engineering subcontractor (Engineer-To-Order (ETO) company). The paper identified 17 issues related to: the market/customer (e.g., rush orders); production process (e.g., routing direction); WLC system (e.g., start-up issues, such as data availability); organisational embedding (e.g., awareness of WLC); and, information flow (e.g., feedback processes). Responses to the issues were presented and areas in need of further research identified. The relevance of the 17 issues to another company has been assessed during the ongoing implementation of WLC described by Huang et al. (2008), where 12 of the 17 issues were particularly significant. However, in some cases the responses were different while Huang et al. (2008) also identified issues not included in the original list, such as uncertainties affecting order progress after release. The need for managerial support and project championing during WLC implementation was also highlighted. This demonstrates the need to collect data on issues affecting WLC implementation from more companies if a broadly applicable strategy is to be developed.

A final key contribution to understanding issues affecting WLC implementation was provided by Perona and Miragliotta (2000) before any of the above four papers were published. Perona and Miragliotta (2000) collected data on company characteristics through interviews with production managers to assess the applicability of WLC simulation results to real-life job shops. Unlike the above, where few companies were considered, Perona and Miragliotta (2000) collected data on 30 companies: 20 SMEs and 10 large organisations. Characteristics considered included: machine features; overall shop features; order features; and demand patterns. The authors noted significant differences between the characteristics of WLC simulations and real-life job shops, questioning the practicality of much WLC research. For example, the authors highlighted practical issues not considered in simulation: large jobs may be split; alternative routings are common; and, shops tend to be larger and more complex in terms of demand, product structures, set-ups, etc. The authors also noted that while WLC research tends to focus on performance in terms of DD adherence, throughput, etc., managers are also interested in issues such as ease of use and robustness. The paper provided a rare glimpse into the characteristics of real-life job shops but did not consider the customer enquiry stage.

\subsection{Assessment of the literature}

Section 2.1 demonstrated that WLC can improve performance when implemented successfully either as a comprehensive solution or when embedded within existing procedures. However, given the intensity of in-depth case or action research, most contributions are based on single cases, meaning the total number of cases is few. Moreover, only limited insight is provided into factors affecting implementation, and scarce attention is given to SMEs. Research reviewed in Section 2.2 considered factors affecting the ability to apply WLC in practice; however, it has either considered too narrow a range of factors or been based on a small number of cases, limiting generality. Nonetheless, taken together, it identifies a wide range of factors that may limit the 
applicability of existing WLC theory and present implementation challenges that must be addressed. WLC is particularly relevant to MTO companies and to SMEs, for which other planning solutions are unsuitable and/or too expensive to implement (e.g., commercially available Advanced Planning and Scheduling (APS) systems). Furthermore, SMEs may present different challenges to the implementation of new concepts such as WLC than large organisations, meaning that they require specific attention.

From the above it follows that further research is required which explores a broad range of factors that affect WLC implementation, including those relevant at the customer enquiry stage, across a larger number of companies but with a particular focus on MTO SMEs.

\section{Research method: cross-sectional case study approach}

This study investigates factors affecting WLC implementation in MTO SMEs. Most previous empirical WLC studies have been conducted over time with a single organisation thereby providing depth but lacking breadth and the external validity and generality of results produced by large samples (Voss et al., 2002). This study represents the 'best-of-both-worlds': it is a cross-sectional study which captures a detailed 'snapshot' of many companies. It begins with the following research questions:

- What characteristics of MTO SMEs that may affect WLC implementation in practice can be identified?

- What are the implications of these characteristics for the development of the WLC concept and strategy for its implementation?

Semi-structured face-to-face interviews have been conducted with 41 companies using an interview protocol, as described below. Each interview was conducted at the company's production facility, providing additional insight into the company's operations.

\subsection{Interview protocol}

An interview protocol consisting of 45 questions was designed to collect consistent data across the cases on a wide range of factors, including those identified in the literature review. The factors are split into the following three groups:

- Customer enquiry and order entry-related issues: important PPC stages for MTO companies yet the former is neglected in much of the literature, e.g., planning at the customer enquiry stage (Stevenson and Silva, 2008).

- Order release-related issues: a fundamental element of most WLC methods. Factors include: production process and product-related issues, as studied by Perona and Miragliotta (2000) and included in the suitability criteria presented by Henrich et al. (2004a), e.g., shop floor configuration and job size and variability.

- Organisational embedding-related issues: important for the implementation of any new initiative and included in comparative case study analysis, such as by Hendry et al. (2008), e.g., awareness of the concept (WLC) and meeting the system data requirements.

Tables 1-3 summarise key factors incorporated in the interview protocol and outline the implications for WLC. An example of a previous study which has identified the issue is provided for each factor.

\subsection{Overview of cases}

Fifty companies were invited to take part in this study and almost all agreed to participate. Eleven of the companies had worked with the researchers on previous projects while a small database of company contact details was also obtained. From this database, 39 companies were selected and 30 agreed to participate. Cases were chosen based on replication logic (Yin, 2003)—all 41 companies were SMEs, as illustrated in Fig. 1; and all produced to-order (for 29, MTO and, for 12, ETO was the dominant production strategy). In other words, all were companies where WLC was expected to be suitable (Henrich et al., 2004a; Stevenson et al., 2005); all make-to-order and some (make and) engineer-to-order. From Fig. 1 it can be seen that three of the companies have more than 250 employees; however, all three satisfy SME criteria for turnover. In other words, all 41 companies have less than or equal to 250 employees and/or a turnover of less than or equal to 50 million Euros.

Interviews were conducted with key personnel in a role of relevance to the implementation of a new PPC concept. The role of interviewees and number of cases were as follows: Managing Director (9 cases); Operations Manager (12 cases); Sales/Customer Enquiry Manager (5 cases); Production and Procurement Planner (10 cases); and Shop Floor Supervisor (5 cases). Where possible, the opinions of the interviewees were validated with quantitative evidence. The companies are active in a wide range of industrial sectors; the most typical are: automotive; rail; electronics; food and beverages; machine tools; chemicals;

Table 1

Customer enquiry and order entry-related issues.

\begin{tabular}{|c|c|}
\hline Issue & Comments \\
\hline $\begin{array}{l}\text { Planning at the customer enquiry stage } \\
\text { (e.g., Stevenson and Silva, 2008) }\end{array}$ & $\begin{array}{l}\text { WLC can support the quotation procedure if its data requirements can be met. Previous attempts to implement } \\
\text { WLC have encountered a lack of formalised planning when quoting for new work. }\end{array}$ \\
\hline $\begin{array}{l}\text { Uncertainty at the customer enquiry stage } \\
\text { (e.g., Stevenson, 2006) }\end{array}$ & $\begin{array}{l}\text { This includes the strike rate or percentage of quotations that become confirmed orders. The time between } \\
\text { submitting a quotation and receiving order confirmation/rejection can also be highly uncertain, affecting other } \\
\text { enquiries. }\end{array}$ \\
\hline $\begin{array}{l}\text { Rush order frequency and handling procedure } \\
\text { (e.g., Stevenson and Silva, 2008) }\end{array}$ & $\begin{array}{l}\text { Rush orders and high-priority jobs impact the performance of WLC, e.g., delaying the adherence of 'normal' } \\
\text { orders. }\end{array}$ \\
\hline Demand variability (e.g., Hendry et al., 2008) & $\begin{array}{l}\text { Where demand is highly variable, the shop load can fluctuate and capacity may be used (for example) on some } \\
\text { MTS items, changing the planning and control problem. }\end{array}$ \\
\hline $\begin{array}{l}\text { Lead times and variability (e.g., Land and Gaalman, } \\
\text { 2009) }\end{array}$ & Includes all factors contributing to the overall delivery lead time of a job. \\
\hline $\begin{array}{l}\text { Communication internally and with customers } \\
\text { (e.g., Silva et al., 2006) }\end{array}$ & $\begin{array}{l}\text { Previous attempts to implement WLC suggest it is becoming important to develop web-based systems that } \\
\text { provide greater interaction with customers. Providing internal access to the system across a network of } \\
\text { computers may also improve internal coordination during the customer enquiry stage (and at later planning } \\
\text { stages). }\end{array}$ \\
\hline
\end{tabular}


Table 2

Order release-related issues.

\begin{tabular}{|c|c|c|}
\hline & Issue & Comments \\
\hline \multirow[t]{6}{*}{ Production characteristics } & Shop floor configuration (e.g., Perona and Miragliotta, 2000) & $\begin{array}{l}\text { A strong dominant flow has a detrimental effect on the } \\
\text { performance of some WLC methods. It may also be necessary to } \\
\text { cater for bottlenecks (whether stationary or 'wandering'). }\end{array}$ \\
\hline & $\begin{array}{l}\text { Assembly operations and routing convergence (e.g., Perona and } \\
\text { Miragliotta, 2000) }\end{array}$ & $\begin{array}{l}\text { Complex product structures with interdependencies between } \\
\text { jobs on the shop floor (e.g., sub- assemblies) can affect the } \\
\text { performance of order release. }\end{array}$ \\
\hline & Order release behaviour (e.g., Huang et al., 2008) & $\begin{array}{l}\text { The performance of order release may be influenced by a wide } \\
\text { range of other factors (than assembly), e.g., disruptions on the } \\
\text { shop floor. }\end{array}$ \\
\hline & Machine characteristics (e.g., Perona and Miragliotta, 2000) & $\begin{array}{l}\text { WLC requires a manageable number of capacity groups; hence, } \\
\text { machines may be grouped into work centres. This depends on } \\
\text { the number and interchange-ability of machines. Machine } \\
\text { characteristics may also lead to sequence dependant set-up } \\
\text { times. }\end{array}$ \\
\hline & Capacity calculations (e.g., Stevenson, 2006) & $\begin{array}{l}\text { WLC requires good estimates of capacity, e.g., to aid release. } \\
\text { Previous attempts to implement WLC have suffered from } \\
\text { difficulties in accurately estimating capacity, or from overly } \\
\text { complex capacity calculations. }\end{array}$ \\
\hline & Output control measures (e.g., Stevenson and Silva, 2008) & $\begin{array}{l}\text { Sufficient flexible output control measures, e.g., subcontracting, } \\
\text { overtime, alternative routings and the reallocation of operators, } \\
\text { are required, e.g., to aid release (and later for expediting jobs on } \\
\text { the shop floor). }\end{array}$ \\
\hline \multirow[t]{3}{*}{ Product characteristics } & Job size and variability (e.g., Henrich et al., 2004a) & $\begin{array}{l}\text { Jobs are usually only released if workload norms will not be } \\
\text { exceeded. When there is a mix of small and large jobs, large } \\
\text { jobs may be delayed as workload lengths may never be low } \\
\text { enough to allow them to be released. }\end{array}$ \\
\hline & Set-up and processing times (ratio) (e.g., Henrich et al., 2004a) & $\begin{array}{l}\text { For WLC to be effective, the total order processing time should } \\
\text { be greater than the set-up time (although short processing } \\
\text { times provide greater workload balancing). }\end{array}$ \\
\hline & Routing lengths (e.g., Henrich et al., 2004a) & $\begin{array}{l}\text { Under the aggregate load oriented WLC method, long shop } \\
\text { floor routings would mean that a job contributes to the } \\
\text { workload of its downstream work centres at the moment of job } \\
\text { release even though it is unlikely to arrive before the next } \\
\text { release decision is made. High routing variability provides } \\
\text { more options for order release and workload balancing. }\end{array}$ \\
\hline
\end{tabular}

Table 3

Organisational embedding-related issues.

\begin{tabular}{|c|c|}
\hline Issue & Comments \\
\hline $\begin{array}{l}\text { Pre-implementation performance } \\
\text { (e.g., Land and Gaalman, 2009) }\end{array}$ & Provides an insight into the performance of companies without WLC. \\
\hline $\begin{array}{l}\text { Awareness of the WLC concept } \\
\text { (e.g., Stevenson and Silva, 2008) }\end{array}$ & $\begin{array}{l}\text { Previous attempts to implement WLC have suffered from a lack of commitment on the part of individuals within the } \\
\text { company; this is partly because practitioners are often previously unaware of the WLC concept. }\end{array}$ \\
\hline $\begin{array}{l}\text { End-user choice and training } \\
\text { (e.g., Hendry et al., 1993) }\end{array}$ & $\begin{array}{l}\text { Previous attempts to implement WLC have suffered from the choice of an ill-informed end-user. The user must gain } \\
\text { sufficient understanding of WLC to use the system effectively. }\end{array}$ \\
\hline $\begin{array}{l}\text { Implementation strategy and support } \\
\text { (e.g., Huang et al., 2008) }\end{array}$ & $\begin{array}{l}\text { It is important to have managerial support, to assign a project champion, to increase the likelihood of successful } \\
\text { implementation. }\end{array}$ \\
\hline $\begin{array}{l}\text { Company technology infrastructure } \\
\text { and planning procedures (e.g., Park } \\
\text { et al., 1999) }\end{array}$ & $\begin{array}{l}\text { WLC may have to interface with other systems and planning methods, e.g., impacting the source of data used by the } \\
\text { WLC system and the expectations of users. }\end{array}$ \\
\hline $\begin{array}{l}\text { Meeting system data requirements } \\
\text { (e.g., Hendry et al., 2008) }\end{array}$ & $\begin{array}{l}\text { The data requirements of the WLC concept must be met. This includes the effective feedback of information from the } \\
\text { shop floor, particularly if continuous release is to be used. }\end{array}$ \\
\hline $\begin{array}{l}\text { Implementation start-up issues } \\
\text { (e.g., Bechte, 1988) }\end{array}$ & $\begin{array}{l}\text { It takes time to get the data in the system up-to-speed and to gain control of the hierarchy of workloads. An iterative } \\
\text { approach to determining many of the parameters underpinning the methodology is often adopted (e.g., maximum } \\
\text { workload lengths). }\end{array}$ \\
\hline
\end{tabular}

aerospace and defence; and industrial equipment. The supply chain positions of the companies were: OEM, close to the endcustomer (13 cases); tier-one supplier (15 cases); tier-two supplier (10 cases); and raw material provider (3 cases).

\section{Cross-case analysis}

The following subsections are organised around Tables 1-3 and discuss characteristics of the 41 MTO SMEs. Each subsection concludes with a brief description of its contribution to the research agenda and implementation strategy summarised in Section 5. Note that the research agenda and implementation strategy have been developed simultaneously. Hence, in response to some of the characteristics encountered, it is recommended in the research agenda that the theory underpinning the WLC concept is moved closer to common practice in MTO SMEs while for other characteristics, it is recommended in the implementation strategy that organisational change takes place to move common practice in MTO SMEs closer to the theory underpinning WLC. The former is recommended where existing processes are 'good' (or appear difficult to change) and fundamental WLC principles will not be undermined; the latter is recommended where fundamental WLC principles are involved. 
a

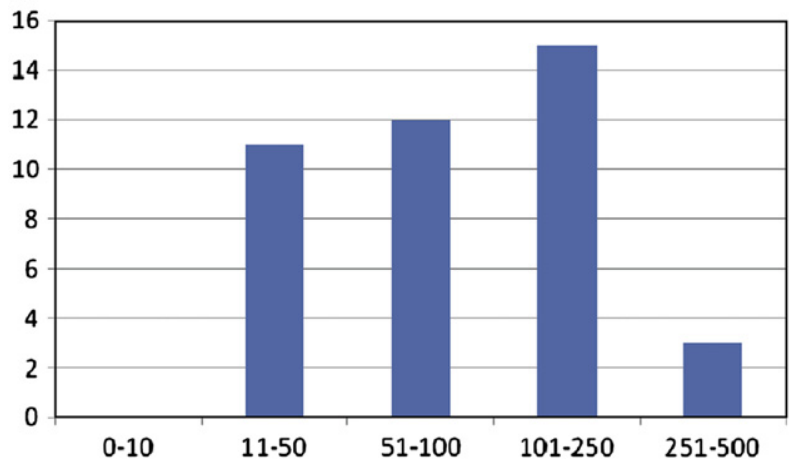

b

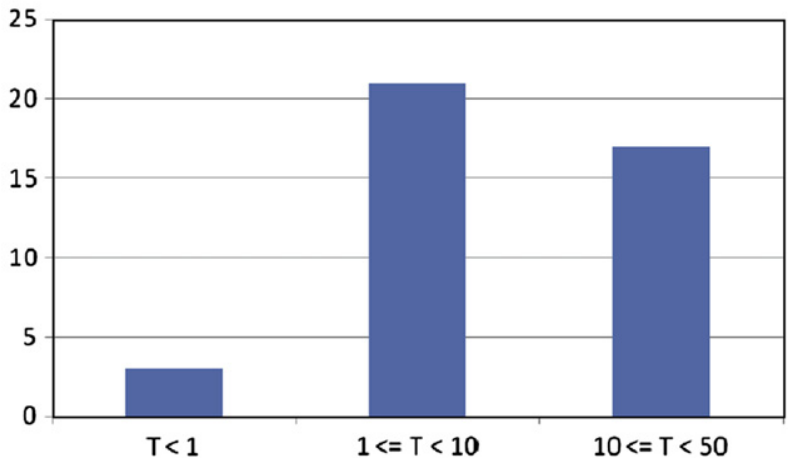

Fig. 1. Company size: (a) number of employees and (b) turnover (T: $£ \mathrm{~m})$.

\subsection{Customer enquiries and order entry-related issues}

A comprehensive WLC concept requires detailed information on customer enquiries, e.g., on processing and set-up times and routings, in order to determine feasible DDs using a formalised planning approach. Therefore, it is important to understand the amount of data routinely available in MTO SMEs prior to order entry. It is argued that this is likely to be influenced by the ratio between new and repeat business; for example, there is a greater incentive to record data at the customer enquiry stage when a large proportion of the workload is repeat business as the data will be reusable. While all 41 companies produce on receipt of a customer order, over half (22 cases) acknowledged that at least $50 \%$ of the order book is made up of repeat orders. Only 4 cases claimed that every order is different. Despite the level of repeat business in many of the cases, little detailed planning currently takes place at the customer enquiry stage. This lack of planning may be explained by the fact that, in 36 cases, customers generally dictate or propose DDs. Only 3 cases claimed that detailed planning takes place to determine realistic DDs; in most cases, only very rough planning takes place. For example, in 13 cases, DDs are set or checked based on experience; in 14 cases, a standard lead time (e.g., 3 weeks) is used when tendering or checking the feasibility of a DD proposed by a customer. In light of this evidence, an implementation strategy should put strong emphasis on training (e.g., so personnel understand the importance of detailed planning at the customer enquiry stage-a core element of a comprehensive WLC concept) and organisational change; however, if this is not possible, research should investigate how the data requirements of WLC at the customer enquiry stage can be reduced without affecting performance. While there may be some scope to renegotiate unrealistic DDs, the emphasis at order entry should be on providing decision support for checking the feasibility of DDs proposed by customers. In other words, on what-if scenario planning and determining how capacity can be redistributed to meet DD requests. This could build on Corti et al. (2006) in which a heuristic based on Kingsman (2000) was presented to support managers in checking DDs proposed by customers.

The customer enquiry stage of WLC assigns a proportion of the workload of prospective orders to the total workload of the shop based on the strike rate or order winning probability (Kingsman and Mercer, 1997), reflecting uncertainty at the customer enquiry stage in terms of order confirmation. Recent research has suggested that when the strike rate is low (e.g., 10\%), and therefore the contribution to the total workload is low, the strike rate can be ignored (Silva et al., 2006; Stevenson, 2006). However, only one company had a strike rate under $10 \%$; in 25 cases, it was claimed to be over $40 \%$, while 4 cases claimed it to be over $80 \%$.
This suggests that including the strike rate percentage is important in practice and underlines why formal planning at the customer enquiry stage should be adopted. In addition, 28 cases suggested that the time between submitting a tender and being notified of acceptance/rejection is highly variable. This explains why, where DDs are negotiable or can be defined by the company, a lead time (e.g., of ' $x$ weeks' from receipt of order confirmation) is often quoted rather than a particular DD. This should be reflected in the type of customer enquiry planning supported by WLC (i.e., a focus on lead times rather than specific DDs).

Research has highlighted problems caused when firms receive rush orders at short-notice with tight DDs. WLC has not been developed to handle rush orders thus they affect the ability to plan and control effectively, with knock-on effects for other jobs (Hendry et al., 2008). In 30 cases, interviewees acknowledged a high rush order arrival rate; a further 7 cases indicated that rush orders occur but not regularly. This was a particularly notable problem for upstream members of supply chains, highlighted by all raw material providers and 8 out of the 10 tier-two suppliers. Interestingly, none of the companies suggested that they would reject a rush order. This suggests that WLC should be refined to improve its ability to handle rush orders while an implementation strategy may require the involvement of customers, e.g., to encourage them to provide suppliers with early-notice of future orders where possible. Hendry et al. (2008) proposed reserving a percentage of capacity for rush orders based on arrival intensity; however, only 4 cases agreed that this would be an effective solution-because the arrival pattern is too unpredictable to know when capacity would be required. The most common approach adopted in practice is to give priority to rush orders ( 28 cases); the second most common is to renegotiate the DDs of other jobs (23 cases; note that many companies use a combination of the two approaches). These two solutions are in line with recent research by Huang et al. (2008) and Thürer et al. (2010). First, Huang et al. (2008) proposed 'impact analysis' whereby the impact of accepting a rush order on other jobs is evaluated, with a view to renegotiating DDs where necessary; however, its effectiveness has not been assessed. Second, through simulation, Thürer et al. (2010) demonstrated that giving priority to rush orders in the pool is an effective solution; however, the solution has not been implemented in practice.

In 33 cases, interviewees indicated that demand is variable/ highly variable, going through significant peaks and troughs. About half of the firms claimed that the current shop load was 'good' (i.e., machines are busy but not overloaded) while about a quarter claimed the shop was overloaded and the rest claimed that the shop was under-loaded. In 17 cases, interviewees indicated that capacity is reduced during periods of under-load (e.g., by shutting down some machines or reducing operator 
hours) while in 16 cases excess capacity is used on stock replenishment orders. Hendry et al. (2008) previously highlighted that in low-demand periods, excess capacity may be used on MTS items, but that this changes the planning and control problem. Clearly, many firms are not "pure MTO" and research should investigate, e.g., through simulation, the impact of a hybrid MTO/ MTS strategy on the effectiveness of WLC. Land et al. (2010) explored how to handle periods of overload to reduce tardiness but periods of under-load should also be considered.

One contributing factor to overall (delivery) lead time, and which relates to the pre-production lead time, was particularly notable in the data: the D\&E stage. In 15 cases, D\&E was a significant contributor to the overall delivery lead time as orders converge on D\&E after order acceptance, as previously identified by Land and Gaalman (2009). As may be expected, this was particularly notable amongst ETO cases. This calls for further research into the conceptual development of WLC to accommodate D\&E. In 12 cases, it was suggested that D\&E delays can be predicted; therefore, one solution may be to create $D \& E$ as a capacity constrained resource: the first work centre in a job's routing, but simulation should investigate the effects of alternative solutions on performance. Land and Gaalman (2009) explained that Bertrand and Muntslag (1993) had in fact previously proposed treating engineering as a separate 'production' unit in the planning framework of a company, but left the detail of this to future research - this remains outstanding.

The final consideration relates to the use of computers and the Internet for internal and external communication during the order process. Only two companies make no regular use of formal communication mechanisms during the process while 26 use a network of PCs for internal communication. While many companies use email to communicate with customers (26 cases) and suppliers (23 cases), only two have websites through which customers can place or track orders. Given that WLC spans tendering, production planning, order release, etc, software should be accessible by multiple internal users but providing access to customers and suppliers is not a key issue at present.

\subsubsection{Contribution to research agenda and implementation strategy}

The customer enquiry and order entry stages are considered key control stages for MTO companies but, in this set of cases, limited planning typically occurs and delivery terms are often dictated by customers, with rush orders common. Future conceptual development of WLC should explore how data requirements at the customer enquiry stage can be simplified whilst still providing good quality support for DD decisions; and how WLC can be developed to best handle rush orders. Greater decision support is also required to aid negotiations with customers and determine how to redistribute capacity to meet tight DDs. Other considerations include how hybrid production can be effectively planned and controlled, how the D\&E stage can be incorporated, and at what point the strike rate percentage becomes significant (and important to incorporate in workload calculations).

Significant training and organisational change is required during the implementation process for formalised customer enquiry management procedures to be successfully adopted in many of these companies. An implementation strategy should emphasise the importance of planning at the customer enquiry stage and of flexible resources to cope with demanding customers at order entry. It should also involve customers to encourage them to share information as early as possible, thereby facilitating more proactive planning. Finally, at the pre-implementation stage, WLC systems should be designed which are accessible to multiple users.

\subsection{Order release-related issues: production process and product characteristics}

\subsubsection{Production process characteristics}

Routing direction (part of shop floor configuration) affects the suitability of WLC order release methods, e.g., the aggregate load approach (Oosterman et al., 2000). Many simulations focus on the pure job shop with random routings (e.g., Land, 2006) but only 2 of the cases can be classified as a pure job shop. In 20 cases, the shop can cope with routing variability but a dominant flow direction generally exists, i.e., a general job shop. In 18 cases, only limited routing variation with a strong dominant flow was identified, i.e., a general flow shop; in 1 case, a pure flow shop was evident. While it is implicit in some WLC research that the main objective is to improve performance in pure job shops, this evidence supports Enns' (1995) argument that configuration is unlikely to lie at one of the extremes, and suggests performance in the general flow and general job shops should be the key criterion.

In 30 cases, interviewees were able to identify a bottleneck within the production process; and in 24 of these cases, the bottleneck changes over time. This again has implications for the choice of WLC release method; for example, if a method which only controls the bottleneck is chosen (see, e.g., Glassey and Resende, 1988; Philipoom et al., 1993; Enns and Prongue-Costa, 2002) it may be susceptible to the 'wandering bottleneck' problem. The data confirms the significance of bottleneck resources and calls for more research into the effects of bottlenecks on the performance of WLC and how bottlenecks can be overcome. Moreover, either decision support is required to assist practitioners in choosing appropriate WLC methods for a given shop floor or robust methods that work well across a range of characteristics should be developed. The latter could use the robustness index introduced by Cigolini et al. (1998).

Most WLC research neglects complex products and how to coordinate release and production between sub-assemblies before convergence on the final assembly stage. The data highlights the significance of product complexity: in 22 cases, the typical product was complex with sub-assemblies. This was particularly notable amongst OEMs (11 out of 13 cases) and tierone suppliers ( 9 out of 15 cases). A key question is whether all parts should be released together or treated independently. Bertrand and van de Wakker (2002) tested the impact of sub-assembly orders on order release finding that performance is improved by releasing all sub-assemblies at the same time compared to treating them independently. More recently, Huang et al. (2008) suggested considering the structure of the end-product as a network: calculate the critical path and focus efforts on the timely release and production of critical path activities; however, the effectiveness of this has not been validated. There is a need to conduct more research into the impact of complex product structures on the performance of WLC and to validate the solution proposed by Huang et al. (2008).

Much WLC research has focused on rational order release (or pool selection) rules in which it is assumed orders are released according to, e.g., earliest DD (e.g., Weng et al., 2008) or planned release date (e.g., Henrich et al., 2006); however, little is known about the release behaviour of planners in practice. Encouragingly, the most important release criterion in the cases is the DD of jobs (considered the most important criterion in 31 cases) but current machine loads are rarely considered (the most important criterion in only 1 case). Overall, the second most important criterion is job size/complexity; third is the importance of the customer; fourth is the availability of materials/tooling; and fifth is the profitability of a job. This suggests that typical release procedures differ from WLC principles and that significant training and organisational change may be required to successfully 
implement WLC at the order release stage. Future research should investigate the influence of release criteria typically adopted in practice on the performance of order release. This could build on Bertrand and van Ooijen (2008) who focused on financial considerations at order release, demonstrating that controlled order release can reduce total costs, such as WIP and late delivery costs. In addition, interviewees also identified issues that affect the release timing of other jobs; most notably, disruptions on the shop floor, i.e., events which slow the progress of orders already on the shop floor meaning other jobs may be held back in the pool for longer than anticipated. These include: a rush order taking priority over another job; a subcontracted operation taking longer than expected; and, production errors leading to rework or the release of an additional production order (to make-up the quantity). Future research should consider the effects of such events after release on the performance of WLC. Furthermore, the reference to subcontract delays highlights the influence of issues beyond the boundaries of the firm on PPC but supply chainrelated issues have been neglected in the WLC literature.

Recent research has begun to investigate other practical issues affecting order release, including the impact of machine characteristics: most notably in the context of sequence dependent setup times (e.g., Kim and Bobrowski, 1995; Fernandes and CarmoSilva, in press) and grouping machines (Henrich et al., 2004b, 2006, 2007). For example, Fernandes and Carmo-Silva (in press) found that release frequency and shop load affect whether local control (at dispatching) leads to better or worse results than central control (at order release) when set-up times are sequence dependent, but only in a pure flow shop. Henrich et al. (2004b) found that feedback requirements from the shop floor to support order release can be reduced by grouping machines into work centres and controlling the work centre load rather than each machine. In 25 cases, sequence dependent set-up times are an important issue while, in 37 cases, machines are grouped into work centres. Groupings are generally based on machine interchange-ability; a secondary consideration is the operator responsible for the machines (machines with common operators may be grouped together). This confirms the importance of these two issues to MTO SMEs but further research is required, e.g., to extend Fernandes and Carmo-Silva's (in press) study to shop conditions more typical of MTO SMEs.

The performance of WLC in practice, including order release, requires a good understanding of capacity and its flexibility. Most WLC simulations assume capacity is evenly distributed across shop floor resources, constant from one day to the next, and constrained by machine hours only; there is little research, for example, into Dual Resource Constrained (DRC) shops. However, in just 10 cases is capacity thought to be constrained by machine hours only; in 13 cases it is constrained by operators; and, in the remaining 18 cases, a combination of labour and machines. This demonstrates that capacity is more sophisticated in MTO SMEs than is typically modelled in WLC simulations and calls for more research into the performance of WLC in DRC shops.

The main output control measures suggested in WLC research are: reallocating operators from under-loaded to overloaded work centres; re-routing jobs; overtime; and subcontracting an operation or job. The first two are generally recommended over the latter two given that they are less costly (Kingsman, 2000). The data suggests overtime is the most commonly used output control measure in practice (used in 37 cases), sometimes at short-notice (23 cases) but sometimes only if agreed far in advance (14 cases). In addition, reallocating operators, re-routing jobs and subcontracting are also commonly applied (in 23, 7, and 14 cases, respectively). Hence, the practice of MTO SMEs is in line with the literature although the focus is on overtime, which is not always as immediately available or flexible as in many simulations. Future research should give more attention to the effects of output control decisions on production costs (e.g., the cost of producing on-time) and the degree of capacity flexibility on the performance of WLC. The latter could revisit the work of Park and Bobrowski (1989) and Bobrowski and Park (1989) which showed that flexible workers have a positive effect on shop floor performance.

\subsubsection{Product characteristics}

Order release performance is affected by job size and variability; for example, when there is a mix of small and large jobs it can be difficult to release large jobs effectively (Silva et al., 2006; Stevenson and Silva, 2008). In the data from the 41 cases, as with the customer enquiry stage, variability also affects order release; for example, there is high variability for the total work content and throughput time of jobs. This confirms that job size variation is common in MTO SMEs and research should investigate its influence on performance. Thürer et al. (2010) found that giving priority to large jobs at order release significantly improves the performance of large jobs with only a minor performance loss for small jobs but field research is required to confirm the effectiveness of this solution in practice. Some processing time variability across jobs at a particular work centre and across work centres for a particular job is also common in the cases. Henrich et al. (2004a) indicated that high variability for the former can be accommodated by WLC but variability in the latter limits workload balancing opportunities. The authors also emphasised the importance of the ratio between processing and set-up times; for WLC to be effective, the total order processing time should be much greater than the set-up time. For 27 of the cases, this criterion is satisfied; in the other 14 cases, there would be a need to reduce set-up times before WLC could be implemented effectively. Note that, in 12 of the 14 cases, interviewees believed that set-up time reduction would be possible.

Finally, most simulations assume jobs only visit a machine once (i.e., no re-entrant loops) and that the number of machines is limited to six, according to the job shop model introduced by Melnyk and Ragatz (1989), or at most 12 (e.g., Cigolini and Portioli-Staudacher, 2002). Hence, the routing length is typically limited to a maximum of six operations. Yet, the case study data indicates that the routing length can be considerably longer. The average typical routing length of a job across the 41 interviews is 11 operations. The majority of typical routing lengths are below 15; however, there is a long tail. While the minimum routing length is 2 , the longest is 28 operations. Future research should explore the performance of WLC order release mechanisms when routing lengths increase.

4.2.2.1. Contribution to research agenda and implementation strategy. The discussion above underlines differences between the production process and product characteristics of MTO SMEs and of simulation models used to test and refine WLC, confirming many of the findings in Perona and Miragliotta (2000). This provides a starting point for developing more realistic models. For example, while many simulations focus on the pure job shop, the general flow and job shops are more typical of MTO SMEs. This has implications for developing more complex and realistic simulation models and developing a robust WLC concept to handle the complexities of real-life production environments, including dual resource constraints, assembly requirements, sequence dependent set-up times, financial considerations, etc. Human factors should also be given greater consideration. The most notable contribution thus far was made by Bertrand and van Ooijen (2002) who concluded that the WIP level influences worker productivity and thus processing times, arguing that WLC can 
maintain WIP at the optimal productivity level. There is scope for further work into human factors, including into the order release behaviour of real-life planners.

WLC is a leading solution for stabilising throughput times; given that many of the companies noted considerable variation in shop floor throughout times; it could be argued that many would benefit from WLC. However, the discussion also highlighted a poor fit with many of the other ideal company characteristics for WLC outlined in Henrich et al. (2004a)_WLC principles would represent a significant change to current order release procedures in many of the cases. In some companies, it may be possible to improve the fit during implementation, e.g., by reducing set-up times, but an implementation strategy should begin by assessing the suitability of WLC and to what extent fit can be improved and then maintained.

\subsection{Organisational embedding-related issues}

It is widely accepted that customers are becoming increasingly demanding in terms of shorter lead times, more customised products, and perhaps most importantly: more reliable delivery performance. In general, (pre-implementation) delivery performance of the companies is poor. In 17 cases, less than $60 \%$ of orders are delivered on-time and, in 6 cases, less than $40 \%$. In only 9 cases was on-time delivery performance over $80 \%$; hence, there is a clear need to improve performance. Therefore, it is argued that many of the companies would benefit from WLC, given that it has the potential to improve delivery performance. However, given that previous attempts to implement WLC have suffered from a lack of commitment towards implementation within the company, it is important to explore other factors that may serve to motivate WLC adoption.

A lack of motivation may be due to low awareness of WLC in practice (Stevenson and Silva, 2008). In the case study data, the most recognised PPC-related concept is lean, including Kanban (27 cases) and the second most is MRP (25 cases) yet neither is considered highly suitable to MTO companies (Stevenson et al., 2005). Ten interviewees claimed to be aware of WLC; however, 7 had taken part in a previous project that introduced them to some WLC principles thereby inflating this figure. Overall, the data supports the claim that WLC is not well recognised by practitioners. To improve the likelihood of successful implementation, awareness of WLC in practice should be increased. Highlighting similarities between WLC and the universally recognised lean philosophy (e.g., variance control: Hopp and Spearman, 2004) may encourage practitioners to engage with the concept.

Interviewees were asked to identify critical success factors for PPC implementations. The most important factors included: strong leadership and championing; selecting an appropriate end-user for the system; clear understanding of the concept within the company; and, regularly monitoring the performance of the project and PPC system. This confirms the importance of issues relevant to the implementation of any new initiative, e.g., project management and performance measurement, and the importance of choosing an appropriate end-user (Hendry et al., 1993) and providing training (Stevenson and Silva, 2008; Stevenson et al., 2009). In addition, top management support followed by anticipated benefits were the two most cited reasons for adopting a new initiative; hence, it is important to gain top management support and ensure key actors are aware of the benefits WLC would bring to them and the company as a whole. Such issues are not described in the WLC literature but are well understood in the wider literature, such as on ERP implementation (e.g., Sumner, 2000; Hong and Kim, 2002; Mandal and Gunasekaran, 2003).
Previous research has demonstrated that WLC may need to be embedded alongside existing formal planning and control procedures within an organisation, e.g., which have only recently become accepted in a company or appear to work well, such as a recently implemented ERP system (Hendry et al., 2008) or Drum-Buffer-Rope approach (Riezebos et al., 2003). However, this is only a key consideration in a minority of cases-in many of the firms, informal processes that could be replaced by WLC are currently used. Many cases rely on a combination of: informal discussions between the operations manager and shop floor supervisor to establish production priorities (23 cases); a notice board to highlight key orders or list WIP (15 cases); and a spreadsheet of orders and DDs to plan and monitor performance (13 cases). Hence, although formally adopting WLC would require significant organisational change, it could replace these methods. However, in 14 cases, MRP is used and, in 6 cases, a state-of-theart ERP system is used, confirming that WLC may need to be embedded within the existing working practices and systems of an organisation. Fandel et al. (1998) reported that LOOR is included in $28 \%$ of commercially available PPC and ERP systems; therefore, research should investigate previous experiences with LOOR and ERP to aid future implementation efforts where WLC and ERP need to be integrated.

Timely feedback of information from the shop floor to the central planning function where order release takes place is important if release decisions are to be made based on up-todate information on the current loads of work centres. If information feedback is slow, periodic release methods must be implemented; continuous release would be infeasible. In 24 cases, information is informally fed-back verbally from operators to the shop floor supervisor back to production planners while many cases also use route cards; 2 cases indicated order progress information is not fed-back until an order has been delivered. Bar coding is used in just 8 cases and RFID in only 1 case. This suggests that, in many companies, the speed of information feedback is unlikely to be quick enough for continuous release methods to be implemented. Given that continuous release methods have been shown to outperform periodic methods (e.g., Hendry and Wong, 1994; Sabuncuoglu and Karapinar, 1999), research should seek to improve the performance of periodic methods or implementation must include considerable investment in technology.

Finally, a key implementation start-up challenge is how to set WLC parameters, particularly workload norms, for two reasons. First, because practitioners may be previously unaware of WLC and unfamiliar with the process of setting WLC norms; and second, because the shop may be overloaded prior to implementation, meaning tight norms can be difficult to introduce. Given that approximately $25 \%$ of the companies are currently overloaded, gaining control may take time. Implementation could therefore begin by setting loose or infinite workload norms during implementation before gradually tightening them over time postimplementation. Only limited research into WLC parameter setting has been conducted (e.g., Perona and Portioli, 1998; Breithaupt et al., 2002; Land, 2006; Thürer et al., 2011); given the lack of awareness regarding WLC previously identified, greater support for practitioners in setting parameters is required.

\subsubsection{Contribution to research agenda and implementation strategy}

This subsection has highlighted a number of factors which must be considered if WLC is to be implemented successfully. Issues relevant to the implementation of any new initiative, such as top management support, are important but also more specific WLC issues, such as how to embed WLC within the established planning procedures of the company, feedback information from the shop floor, and set appropriate parameters, e.g., workload 
norms. This confirms the relevance of many of the findings in Hendry et al. (2008) to a larger number of companies. Training is also required to educate users of WLC systems; this could build on the training tools provided by Wiendahl et al. (1995) and Stevenson et al. (2009).

\section{Summary of research agenda and implementation strategy}

The above evidence arguably suggests a misalignment between theory and practice, partly explaining why implementation is so challenging; hence, there is much scope for improving the fit between MTO SMEs and WLC. But this is not a one-way relationship: the concept can be refined closer to the needs of practitioners but organisational change may also be required. An agenda for developing the WLC concept is summarised below before Section 5.1 outlines an initial high level implementation strategy, incorporating elements of organisational change:

- Customer enquiry stage: Many of the companies would struggle to adopt the customer enquiry management practices of WLC. Research should explore how data requirements at the customer enquiry stage can be reduced whilst still providing realistic delivery lead time estimates.

- Order entry: For many companies, delivery terms are dictated by customers while short-notice rush orders are common. Greater decision support should be provided for negotiations with customers, e.g., to assess the feasibility of DDs proposed by customers and the impact of rush orders with tight DDs on other jobs, identifying where possible how capacity can be adjusted to improve performance.

- Design and Engineering: D\&E can be a significant contributor to the overall delivery lead time. Research should assess how D\&E activities can be incorporated within the concept, both when quoting lead times at the customer enquiry stage and beyond.

- Order release: The current workloads of resources are rarely considered when planners make release decisions in practice. Research should investigate how the release behaviour of planners in practice influences the performance of WLC order release methods, e.g., considering financial aspects of orders and customer importance. The influence of sub-assemblies, hybrid production strategies, sequence dependent set-up times and shop floor disruptions on order release should also be given greater attention. In doing so, robustness to shop floor configuration should be considered.

Conceptual developments proposed in response to the above can be initially tested through simulation. While we acknowledge that simple models make it easier to diagnose the influence of specific factors on performance, the characteristics of real-life MTO SMEs (e.g., flow characteristics, routing lengths, resource constraints, etc) should be considered in the design of these experiments in order to bridge the gap between theory and practice.

\subsection{An implementation strategy for WLC}

To successfully implement WLC in practice it is necessary to consider three stages: the initial pre-implementation stage; the implementation process; and post-implementation. Note that the third stage (post-implementation) was not considered in the steps specific to LOMC outlined by Wiendahl (1995). Each is outlined below before an overall strategy is summarised in Fig. 2. This strategy provides an overview of the implementation process for WLC; it is a starting point for the development of a more detailed roadmap to support WLC implementation.

(1) Pre-implementation (diagnosis phase): The fit between the company and concept should be assessed, e.g., using criteria outlined in Henrich et al. (2004a). This may also involve evaluating the current soft/hardware infrastructure and developing WLC software to support implementation. Commitment to the project, current business processes and prior logistic performance should also be considered.

(2) Implementation process (theory and practice alignment): If implementation is to go ahead, this should include closing gaps between theory and practice. Organisational change may include grouping machines into a manageable number of work centres (based on interchange-ability), improving the flow of information (e.g., bar code scanners) and reducing setup times. Appropriate aspects of the concept should be selected and configured; for example: order release mechanisms (considering, e.g., bottlenecks, flow direction, etc.) and

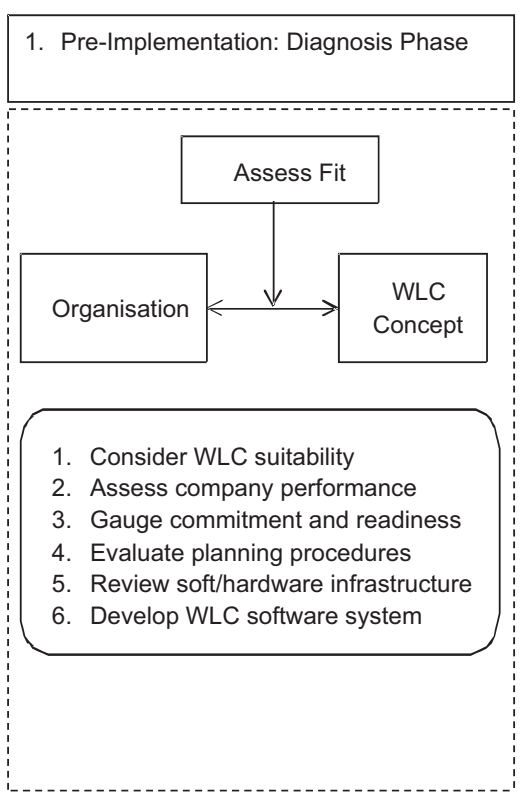

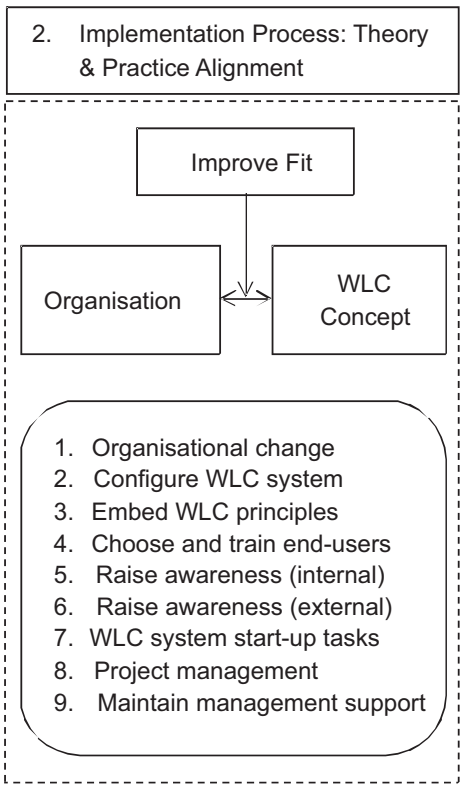

Fig. 2. Implementation strategy for WLC.

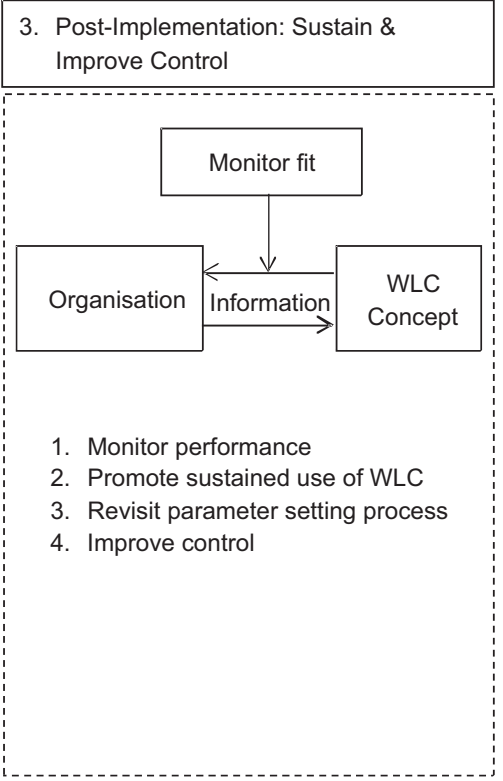


initial parameters (e.g., capacity estimates, output control measures, and workload norms). WLC principles may be embedded within existing procedures (e.g., ERP, DBR, etc.) while appropriate end-users must be chosen, trained and given access to the system. Raising awareness and training employees at all levels of the organisation must be undertaken; where possible, this should be extended to customers. The WLC system must be brought 'up-to-speed' with the current order book and shop floor status. Strong leadership and project management skills are required throughout.

(3) Post-implementation (sustain and improve control): This must focus on: monitoring the performance of the company and WLC system in terms of WIP, throughput, throughput times, ease of use, etc.; sustaining use of WLC over time; and revisiting parameters as appropriate, e.g., to tighten workload norms or adjust capacity estimates.

\section{Conclusion}

It has been argued in the literature that Workload Control (WLC) is of particular relevance to Make-To-Order (MTO) companies and Small and Medium sized Enterprises (SMEs) (Henrich et al., 2004a; Stevenson et al., 2005; Land and Gaalman, 2009). Yet few successful implementations of WLC in MTO SMEs have been reported. This paper contributes to the available literature by highlighting characteristics of MTO SMEs that may affect the implementation of WLC in practice based on evidence collected through semi-structured interviews with 41 companies. In doing so, it builds on comparative WLC case study analysis (e.g., by Hendry et al., 2008) and WLC implementation procedures (e.g., by Wiendahl, 1995),

It is concluded that WLC has the potential to improve the PPC practices of MTO SMEs, including many of the cases described in this paper; however, it is also clear that there is much scope for further research in order to improve the fit and successful implementation of WLC in practice. The data paints a complex picture of MTO production and suggests that research is likely to be required to move theory closer to practice and organisational change to move practice closer to theory. The former is recommended where processes perform well or appear difficult to change; the latter is recommended where fundamental WLC principles are involved. Elements of the WLC concept that can be refined to improve alignment between theory and practice covering the customer enquiry, order entry, design and engineering and order release stages - have been identified; and an initial strategy for the implementation of WLC - covering preimplementation, implementation and post-implementation stages - has been proposed. Given the wide range of characteristics identified, it is argued that, for a WLC concept to be effective, it should be robust to different conditions but also flexible so that appropriate elements can be selected and embedded within existing 'good' company practices.

Three key future research directions emerge from this study. First, although simple models enable the performance effects of individual factors to be diagnosed, the characteristics of MTO SMEs identified should be used to develop more realistic simulations for testing conceptual developments. Second, while data on more companies than in previous WLC studies has been presented, future research could condense the structured interview protocol and use it as the basis for collecting an even larger data set on the characteristics of MTO SMEs through survey research. Statistical analysis could then be performed to validate the findings of this study. And, third, field research should apply and extend the initial implementation strategy presented in order to develop a more detailed roadmap for successful WLC implementation in practice.

\section{References}

Bechte, W., 1988. Theory and practise of load-oriented manufacturing control. International Journal of Production Research 26 (3), 375-395.

Bechte, W., 1994. Load-oriented manufacturing control just-in-time production for job shops. Production Planning and Control 5 (3), 292-307.

Bertrand, J.W.M., Muntslag, D.R., 1993. Production control in engineer-to-order firms. International Journal of Production Economics 30-31, 3-22.

Bertrand, J.W.M., van de Wakker, A.M., 2002. An investigation of order release and flow time allowance policies for assembly shops. Production Planning and Control 13 (7), 639-648.

Bertrand, J.W.M., van Ooijen, H.P.G., 2002. Workload based order release and productivity: a missing link. Production Planning and Control 13 (7), 665-678.

Bertrand, J.W.M., van Ooijen, H.P.G., 2008. Optimal work order release for maketo-order job shops with customer order lead-time costs, tardiness costs and work-in-process costs. International Journal of Production Economics 116, 233-241.

Bobrowski, P.M., Park, P.S., 1989. Work release strategies in a dual resource constrained job shop. Omega 17 (2), 177-188.

Breithaupt, J.W., Land, M.J., Nyhuis, P., 2002. The workload control concept: theory and practical extensions of load oriented order release. Production Planning and Control 13 (7), 625-638.

Cigolini, R., Portioli-Staudacher, A., 2002. An experimental investigation on workload limiting methods with ORR policies in a job shop environment. Production Planning and Control 13 (7), 602-613.

Cigolini, R., Perona, M., Portioli, A., 1998. Comparison of order review and release techniques in a dynamic and uncertain job shop environment. International Journal of Production Research 36 (11), 2931-2951.

Corti, D., Pozzetti, A., Zorzini, M., 2006. A capacity-driven approach to establish reliable due dates in a MTO environment. International Journal of Production Economics 104, 536-554.

Enns, S.T., 1995. An integrated system for controlling shop loading and work flows. International Journal of Production Research 33 (10), 2801-2820.

Enns, S.T., Prongue Costa, M., 2002. The effectiveness of input control based on aggregate versus bottleneck workloads. Production Planning and Control 13 (7), 614-624.

Fandel, G., Francois, P., Gubitz, J.M., 1998. PPS- und integrierte betriebliche Softwaresysteme Grundlagen, Methoden, Marktanalyse. Springer Verlag, Berlin, Heidelberg, New York.

Fernandes, N.O., Carmo-Silva, S. Order release in a workload controlled flow-shop with sequence-dependent set-up times. International Journal of Production Research, in press.

Fry, T.D., Smith, A.E., 1987. A procedure for implementing input/output control: a case study. Production and Inventory Management Journal 28 (4), 50-52.

Glassey, C.R., Resende, M.G., 1988. Closed-loop job release control for VLSI circuit manufacturing. IEEE Transactions on Semiconductor Manufacturing 1, 36-46.

Hendry, L.C., 1989. A decision support system to manage delivery and manufacturing lead times in make to order companies, PhD Thesis, Lancaster University, Lancaster, UK.

Hendry, L.C., 2006. Workload control: for effective integration of SMEs in the supply chain. In: Proceedings of the International Workshop on Successful Strategies in Supply Chain Management, Hong Kong, 17-23.

Hendry, L.C., Wong, S.K., 1994. Alternative order release mechanisms: a comparison by simulation. International Journal of Production Research 32 (12), 2827-2842.

Hendry, L.C., Elings, P., Pegg, D., 1993. Production planning for an artist's studioa case study. European Journal of Operational Research 64, 12-20.

Hendry, L.C., Land, M.J., Stevenson, M., Gaalman, G., 2008. Investigating implementation issues for workload control (WLC): a comparative case study analysis. International Journal of Production Economics 112, 452-469.

Henrich, P., Land, M.J., Gaalman, G., 2004a. Exploring applicability of the workload control concept. International Journal of Production Economics 90, 187-198.

Henrich, P., Land, M.J., Gaalman, G., Van der Zee, D.-J., 2004b. Reducing feedback requirements of workload control. International Journal of Production Research 42 (24), 5235-5252.

Henrich, P., Land, M.J., Gaalman, G., 2006. Grouping machines for effective workload control. International Journal of Production Economics 104 (1), 125-142.

Henrich, P., Land, M.J., Gaalman, G., 2007. Semi-interchangeable machines: implications for workload control. Production Planning and Control 18 (2), 91-104.

Hong, K.K., Kim, Y.G., 2002. The critical success factors for ERP implementation: an organisational fit perspective. Information and Management 40, 25-40.

Hopp, W.J., Spearman, M.L., 2004. To pull or not to pull: what is the question? Manufacturing and Service Operations Management 6 (2), 133-148.

Huang, Y., Stevenson, M., Hendry, L.C., 2008. Organizational embedding of a workload control concept: an action research project. In: Proceedings of the 15th European Operations Management Association (EurOMA) Conference, Groningen, The Netherlands, Conference Proceedings.

Kim, S.J., Bobrowski, P.M., 1995. Evaluating order release mechanisms in a job shop with sequence-dependent set-up times. Production and Operations Management 4 (2), 163-180.

Kingsman, B.G., 2000. Modelling input-output workload control for dynamic capacity planning in production planning systems. International Journal of Production Economics 68 (1), 73-93. 
Kingsman, B.G., Mercer, A., 1997. Strike rat matrices for integrating marketing and production during the tendering process in Make-to-Order subcontractors. International Transactions in Operational Research 4 (1), 251-257.

Kingsman, B.G., Hendry, L.C., Mercer, A., De Souza, A., 1996. Responding to customer enquiries in make-to-order companies: problems and solutions. International Journal of Production Economics 46-47, 219-231.

Land, M.J., 2006. Parameters and sensitivity in workload control. International Journal of Production Economics 104 (2), 625-638.

Land, M.J., Gaalman, G.J.C., 1996. Workload control concepts in job shops: a critical assessment. International Journal of Production Economics 46-47, 535-538.

Land, M.J., Gaalman, G.J.C., 1998. The performance of workload control concepts in job shops: improving the release method. International Journal of Production Economics 56-57, 347-364.

Land, M.J., Gaalman, G.J.C., 2009. Production planning and control in SMEs: time for change. Production Planning and Control 20 (7), 548-558.

Land, M., Su, N.P.B., Gaalman, G., 2010. In search of the key to delivery improvement. In: Proceedings of the Sixteenth International Working Seminar on Production Economics, Innsbruck, Austria, March 1-5, 2, pp. 297-308.

Mandal, P., Gunasekaran, A., 2003. Issues in implementing ERP: a case study. European Journal of Operational Research 146, 274-283.

Melnyk, S.A., Ragatz, G.L., 1989. Order review/release: research issues and perspectives. International Journal of Production Research 27 (7), 1081-1096.

Oosterman, B., Land, M.J., Gaalman, G., 2000. The influence of shop characteristics on workload control. International Journal of Production Economics 68 (1), 107-119.

Park, C., Song, J., Kim, J., Kim, I., 1999. Delivery date decision support system for the large scale make to order manufacturing companies: a Korean electric motor company case. Production Planning and Control 10 (6), 585-597.

Park, P.S., Bobrowski, P.M., 1989. Job release and labour flexibility in a dual resource constraint job shop. Journal of Operations Management 8 (3), 230-249.

Perona, M., Miragliotta, G., 2000. Workload control: a comparison of theoretical and practical issues through a survey in field. In: Proceedings of the 11th International Working Seminar on Production Economics, Igls, Innsbruck, Conference Proceedings, pp. 1-14.

Perona, M., Portioli, A., 1998. The impact of parameter setting in load oriented manufacturing control. International Journal of Production Economics 55, 133-142.

Philipoom, P.R., Malhotra, M.K., Jensen, J.B., 1993. An evaluation of capacity sensitive order review and release procedures in job shops. Decision Sciences 24 (6), 1109-1133.

Riezebos, J., Korte, G.J., Land, M.J., 2003. Improving a practical DBR buffering approach using workload control. International Journal of Production Research 41 (4), 699-712.

Sabuncuoglu, I., Karapinar, H.Y., 1999. Analysis of order review/release problems in production systems. International Journal of Production Economics 62, 259-279.

Silva, C., Roque, L., Almeida, A., 2006. MAPP_A web-based decision support system for the mould industry. Decision Support Systems 42 (2), 999-1014.

Soepenberg, G.D., Land, M., Gaalman, G., 2008. The order progress diagram: a supportive tool for diagnosing delivery reliability performance in make-to- order companies. International Journal of Production Economics 112, 495-503.

Stevenson, M., 2006. Refining a workload control (WLC) concept: a case study. International Journal of Production Research 44 (4), 767-790.

Stevenson, M., Hendry, L.C., 2007. Improving supply chain integration using workload control concept and web-functionality. Production Planning and Control 18 (2), 142-155.

Stevenson, M., Silva, C., 2008. Theoretical development of a workload control methodology: evidence from two case studies. International Journal of Production Research 46 (11), 3107-3131.

Stevenson, M., Hendry, L.C., Kingsman, B.G., 2005. A review of production planning and control: the applicability of key concepts to the make to order industry. International Journal of Production Research 43 (5), 869-898.

Stevenson, M., Huang, Y., Hendry, L.C., 2009. The development and application of an interactive end-user training tool: part of an implementation strategy for workload control. Production Planning and Control 20 (7), 622-635.

Sumner, M., 2000. Risk factors in enterprise-wide/ERP projects. Journal of Information Technology 15, 317-327.

Tatsiopoulos, I.P., 1983. A microcomputer-based interactive system for managing production and marketing in small component manufacturing firms using a hierarchical backlog control and lead time management technology, $\mathrm{PhD}$ Thesis, Lancaster University, Lancaster, UK.

Thürer, M., Silva, C., Stevenson, M., 2010. Workload control release mechanisms from practice back to theory building. International Journal of Production Research 48 (12), 3593-3617.

Thürer, M., Silva, C., Stevenson, M., 2011. Optimising workload norms: the influence of shop floor characteristics on setting workload norms for the workload control concept. International Journal of Production Research 49 (4), $1151-1171$.

Thürer, M., Stevenson, M., Silva, C. Three decades of workload control research: a systematic review of the literature, International Journal of Production Research, in press.

Voss, C., Tsikriktsis, N., Frohlich, M., 2002. Case research in operations management. International Journal of Operations and Production Management 22 (2), 195-219.

Weng, M.X., Wu, Z., Qi, G., Zheng, L., 2008. Multi-agent-based workload control for make-to-order manufacturing. International Journal of Production Research 46 (8), 2197-2213.

Wiendahl, H.P., 1995. Load oriented manufacturing control. Springer Verlag, Berlin Heidelberg, New York.

Wiendahl, H.P., Gläßner, J., Petermann, D., 1992. Application of load-oriented manufacturing control in industry. Production Planning and Control 3 (2) 118-129.

Wiendahl, H.P., Scholtissek, P., Fastabend, H., 1995. Simulation-based training systems for job shop control (TRAIN-F). In: Riis, J.O. (Ed.), Simulation games and learning in production management. Chapman and Hall, London.

Yin, R.K., 2003. Case study research: Design and methods, 3rd Edition Sage Publications, London. 